\title{
Convergent and divergent perspective
}

\author{
Jan B Derȩgowski, Denis M Parker
}

Department of Psychology, King's College, University of Aberdeen, Aberdeen AB9 1FX, UK Received 27 March 1991, in revised form 5 September 1991

Abstract. Use of divergent (or inverse) perspective in pictures is often regarded as arbitrary or even as erroneous in spite of the fact that entire schools of art exist in which this kind of perspective is regularly used.

An experiment is reported which shows that a significant trend towards divergent perspective is experienced by subjects viewing laterally displaced three-dimensional arrays. Centrally viewed arrays show the expected perceptual convergence. It is therefore argued that divergent perspective, under appropriate conditions, is as perceptually legitimate an experience as convergent perspective.

\section{Introduction}

Gillam (1981) deplores the fact that illustrations in various psychological texts purporting to be drawn in perspective are not in fact so drawn. She acknowledges that such deviations may be unimportant as far as artists are concerned but maintains that it behoves the perceptionists not to be cavalier about the matter and concludes that "wrongly drawn perspective ... fails to produce good impressions of the third dimension". She thus implies that perspective [meaning scientific perspective, as defined in Osborne (1981)], is naturally correct and its violations, even when enhancing the aesthetic value of representations, decrease their verisimilitude.

This claim may be erroneous. The works of highly regarded architectural painters such as Pieter Saenredam (Kemp 1984; Derȩgowski 1989, 1990; Parker and Derȩgowski 1990) and Job Berckheyde (Derȩgowski 1989, 1990; Parker and Derȩgowski 1990) show considerable systematic deviations from perspective. These deviations do not appear to be purely aesthetic for when they are corrected [as, for example in Carter's (1967) correction of Saenredam's Grote Kerk] to accord with the rules of perspective the pictures in question do not merely look less pleasing--they look wrong.

Deviations from scientific perspective have also been observed in the real, threedimensional world. Bartel (1958; see Parker and Derȩgowski 1990) has shown that subjects required to draw objects distributed in space, or equispaced objects in a row, show mutually consistent deviations from 'true' perspective. Furthermore it has been shown (ten Doesschate and Kylstra 1955; ten Doesschate 1964) that when observers are required to judge the apparent convergence of luminous tubes placed parallel to their sagittal planes they do not report them as converging at a single point. The more laterally displaced tubes appear to converge at a point further away than do the less laterally displaced ones. In order to obtain a single point of apparent convergence one has to set the tubes so that the more laterally displaced ones are sloping towards the line of sight at a less acute angle to the observer's sagittal plane than are the less laterally displaced tubes. It follows, then, that when viewing the real world there is no single perceptual point of apparent convergence through which all parallel lines appear to pass.

There are therefore two interesting aspects of perspective: (i) not all of its expressions are equally acceptable perceptually when incorporated into paintings; and (ii) the eye does not always see the three-dimensional world in accordance with the 
rules of perspective, and there appears to be concordance between deviation from the rules of perspective applicable to pictures and those applicable to the 'real world'.

The second of the above observations suggests that certain violations of perspective in pictures may not only be aesthetically but also perceptually justifiable; but it may be that some violations, although perceptually justifiable, may, as a result of cultural experience, be seen by observers as being wrong.

Consider the phenomenon of the less rapid convergence of the more laterally displaced lines which is explicable, as shown by ten Doesschate (1964, page 137), in terms of the angles which are supported at the eye by equal parallel segments. Figure 1 illustrates this assertion. The rate of convergence of any two parallel lines corresponds to the ratio of angles which corresponding points on those lines support at the eye $(\mathrm{O})$. Thus the rate of convergence of lines $\mathrm{AD}$ and $\mathrm{BE}$ is given by the ratio of angle $a$ to angle $b$, and the rate of convergence of lines $B E$ and $C F$ by the ratio of angle $c$ to angle $d$. As $a: b$ is less than $c: d$, lines $\mathrm{AD}$ and $\mathrm{BE}$ appear to converge more rapidly than do lines $\mathrm{BE}$ and $\mathrm{CF}$. Now, it is clear that if a plane of projection is interposed between the viewer and the scene a perspective view of the lines will be obtained in which central and lateral pairs of lines will indeed converge. Nevertheless, it should be emphasised that it is the ratio of the visual angles that drives the viewer's perception of convergence, as shown by ten Doesschate (1964), not the principles of scientific perspective (ie what would be obtained on the projection plane).

Extension of this reasoning, illustrated in figure 2, has interesting implications for the most blatant violation of conventional linear perspective in pictures, the so-called inverse perspective, wherein receding lines are shown as diverging. In this figure the

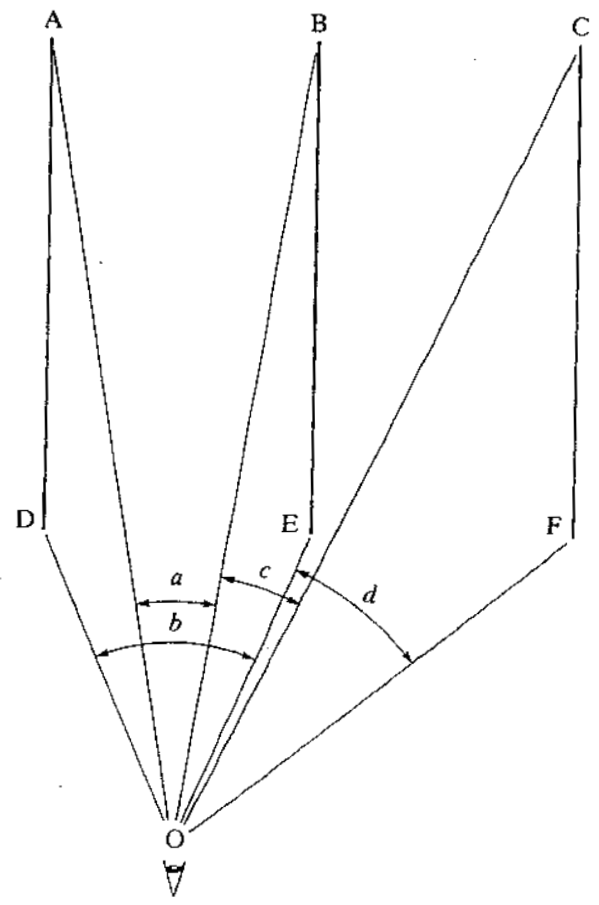

Figure 1. Angles supported at the eye $(\mathrm{O})$ by three parallel segments, $A D, B E$, and $C F$. It is apparent that the ratio of angles $a: b$ is smaller than the ratio $c: d$, and lines $\mathrm{AD}$ and $\mathrm{BE}$ are seen (as ten Doesschate has demonstrated) as converging more rapidly than the lines $\mathrm{BE}$ and $C F$. 
eye is represented by point $\mathrm{O}$, and the two identical rectangles represent two identical rectangular objects. The ratio of the angles made by the projection lines clearly shows that, whereas in the case of the rectangle nearer to the eye the nearer of the short edges supports a larger angle than its further counterpart, the opposite is true in the case of the further rectangle. This suggests that, whereas the former should be seen as converging, and therefore in accord with the rules of perspective, the latter should be seen as diverging and therefore should be drawn in inverse perspective. This phenomenon should be observable even under conditions of much decreased information wherein a rectangle is reduced to four points representing the four corners (Attneave 1954).

Divergent drawings are indeed often made by children and are characteristic of certain traditional schools of art. But the use of inverse perspective is not confined to the unsophisticated or the esoteric. Several authors report observing inverse perspective in drawings of very sophisticated students (see Parker and Derȩgowski 1990), and inverse perspective can be found in Durer's earlier woodcuts, for example in his portrayal of a book in his illustration, St.Jerome in his Study executed in about 1492 [see figure 22 in Kurth (1927)]. It may be that inverse perspective is experienced daily by observers, just as ten Doesschate's experiments show that nonconvergence of parallels is, but that it is simply not taken account of because of the preference of draughtsmen to depict receding lines as convergent. This argument has been put forward by Parker and Derȩgowski (1990), who quote supporting evidence from experiments by Zajac (1961) that show that within a certain, admittedly narrow, range of circumstances a right rectangular parallelopiped placed in front of an observer is seen as having divergent sides.

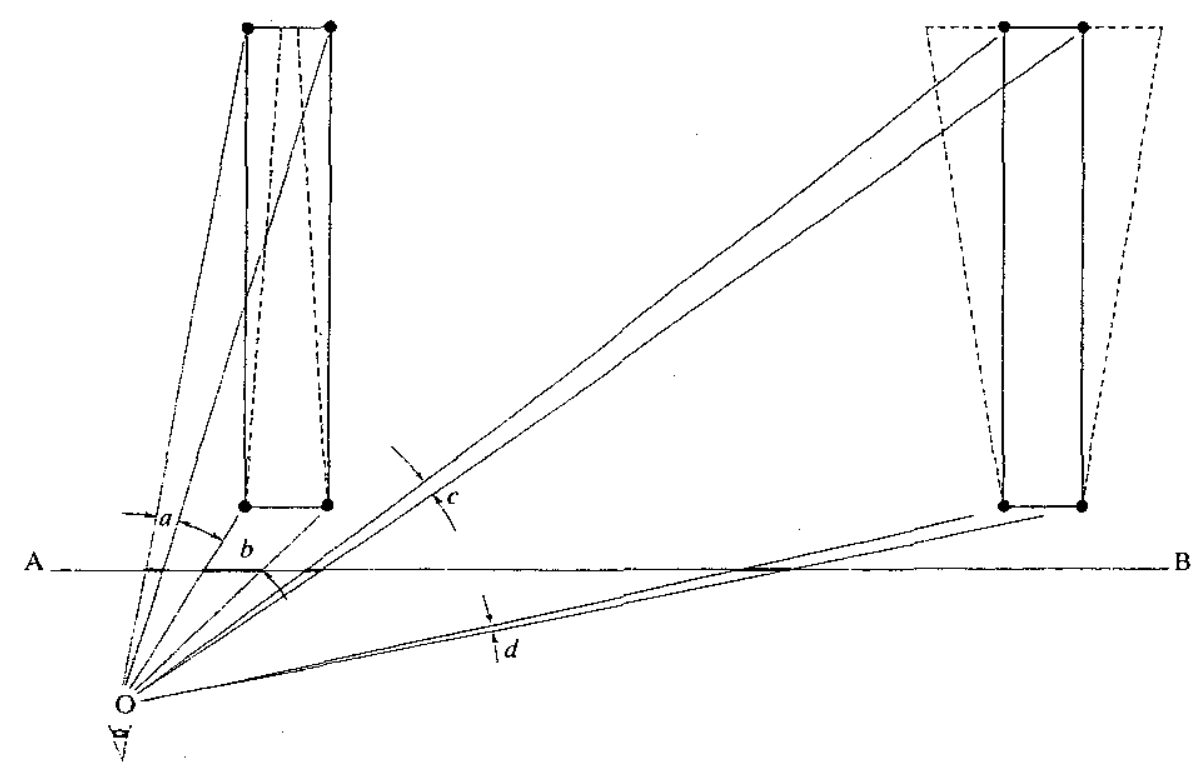

Figure 2. Projections of two identical rectangles at the eye $(\mathrm{O})$. The ratios of angles, $a: b$ and $c: d$, show that whilst the frontally presented rectangle should appear to be converging, the laterally presented rectangle should appear to be diverging. The dotted outlines represent figures the length of whose sides are such that they are in the same ratio as are the corresponding angles. Line $\mathrm{AB}$ shows a position of a hypothetical picture plane. It is apparent that on this surface the intercepts made by the rays drawn to the more distant elements are consistently shorter than those drawn to the less distant ones. 
The experiment reported below is intended to investigate the perception of parallel lines under more general circumstances than those used in Zajac's experiment, in order to find out whether, as the above discussion suggests, there is a broader experiential basis for divergent perspective than that provided by Zajac's study.

\section{Method}

\subsection{Subjects}

Twelve adult subjects, five men and seven women, were drawn from the Department's panel. They were drawn at random, as far as their availability permitted, and were required to state if they had any known ocular or perceptual defects. Subjects who declared defects, other than the use of spectacles, were excluded from the experiment.

\subsection{Apparatus and procedure}

The layout of the apparatus is shown in figure 3. The apparatus consisted of two mobile lights, $8 \mathrm{~mm}$ in diameter, and two identical fixed lights. The two sets of lights were placed on the laboratory floor in parallel lines, $3 \mathrm{~m}$ apart. A third parallel line, $1.25 \mathrm{~m}$ distant from the line of fixed lights and $4.25 \mathrm{~m}$ distant from that of the mobile lights, was drawn on the laboratory floor. On this line two stances for the subject were marked, one on the perpendicular bisector of the distance between the two fixed lights, and the other at a distance of $3 \mathrm{~m}$ from this position.

The apparatus was so arranged that by moving an appropriate hand-held joystick each of the mobile lights could be moved independently along the straight line on which they lay. The range of this movement was restricted so that at their closest the lights could be $0.08 \mathrm{~m}$ apart and at their most distant $1.8 \mathrm{~m}$ apart. The lights moved slowly and steadily when the joystick was pushed. The two fixed lights were $1 \mathrm{~m}$ apart. The experiment was carried out in a darkened laboratory so that the mobile lights shone brightly against a dimly visible floor.

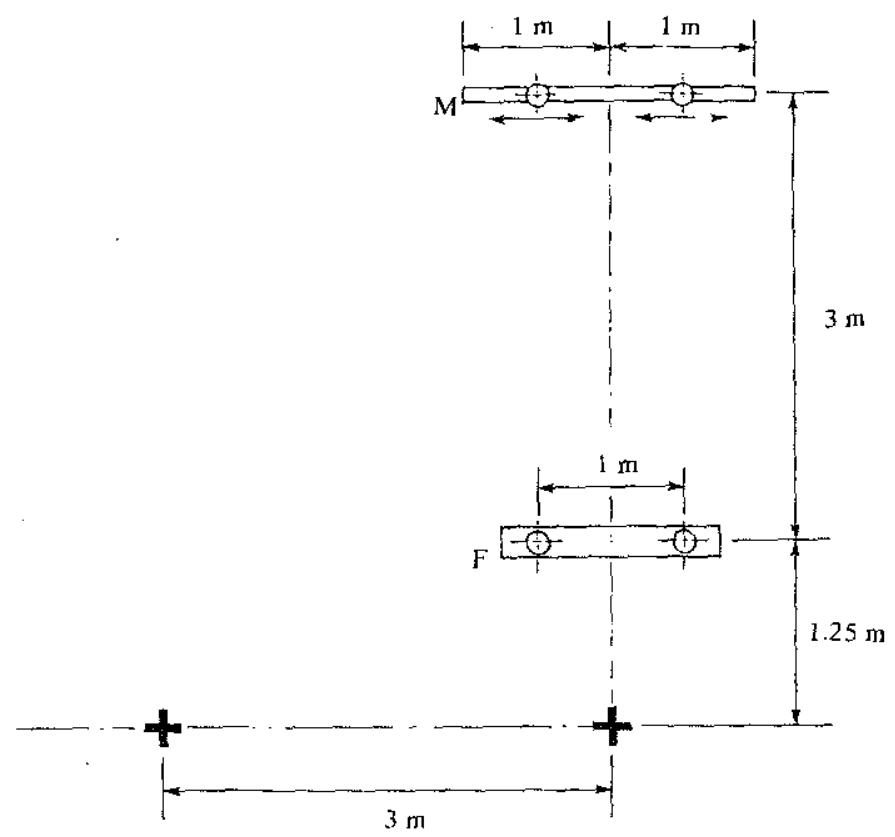

Figure 3. Subjects were required to set the two mobile lights $(\mathbf{M})$ so that they, together with the fixed lights $(F)$, formed a 'real rectangle'. The two crossed indicate the two stances in which the subjects stood. 
Subjects were first allocated, in alternation, to either of the two stances, and were instructed how to operate the joysticks. They were entirely unconstrained in their eye and head movements when making their responses but were not allowed to move from their stances. The experimental conditions approximated closely to those of 'normal' viewing. Subjects were instructed to set the two mobile lights so that, together with the two fixed lights, they formed corners of a 'real rectangle'. Each subject adjusted the positions of the mobile lights ten times, five occasions beginning with the lights at their furthest apart, and five beginning with the lights at their closest together. All the final settings made were recorded.

Each subject was then required to move to the other stance and the procedure was repeated.

\section{Results}

The mean distances apart at which each subject set the two mobile lights were calculated for each of the two stances. In all cases the mean obtained at the frontal stance was greater than that obtained from the laterally displaced stance. Furthermore, all but two of the responses made from the frontal stance were greater than the distance of $1 \mathrm{~m}$ at which the fixed lights were placed, and all but two responses made from the laterally displaced stance were less than $1 \mathrm{~m}$. A $t$-test was used to compare the settings in the two stances with the correct setting of $1 \mathrm{~m}$. The results were as follows: frontal stance: mean setting $-1.05 \mathrm{~m}, t=3.22, p<0.003$; laterally displaced stance: mean setting $-0.76 \mathrm{~m}, t=2.89, p<0.006$. Comparison of the responses made in the two stances (mean difference, $0.29 \mathrm{~m}$ ) showed that the difference was also significant $(t=2.66, p<0.02)$.

Therefore responses made from the two stances differ radically. Frontally viewed distances are overestimated, and laterally viewed distances are underestimated.

\section{Discussion}

The results show that the highly constraining circumstances used by Zajac (1961) to show that parallel lines can be perceived as divergent are not the only ones under which the phenomenon can occur. Such percepts can arise under much more readily encountered, and therefore more generally experienced, situations. This calls into question the notion that the divergent perspective which occurs in the drawings of some artists and in some schools of art is an esoteric convention which they have adopted and which is contrary to the 'natural' inclinations of the eye; on the contrary, it shows divergent perspective to be as legitimately derived from perceptual experience as is the more common convergent perspective of present day 'Western' pictures. The argument put forward by Parker and Derȩgowski (1990) suggesting that divergent perspective is as legitimate a representation of experience of, under appropriate circumstances, space, as is the convergent perspective, is thus supported.

The results do not indicate, however, the mechanism wherewith the artist translates this experience in order to convey it on the flat surface on which he or she is composing a picture. Clearly, as the segments marked on line $\mathrm{AB}$ in figure 2 indicate, if the artist were drawing as if the plane of the picture were parallel to the shorter edges of the rectangles shown in the figure and the plane intersected the rays drawn in that figure, then divergent perspective could not occur since the passage of rays extending to the two more distant edges would always make shorter intercepts on the picture plane than would those made by the rays extending to the two nearer edges, and the ratio of the corresponding intercepts would remain constant. Therefore, if the picture were conceived as a window pane the kind of divergent perspective investigated could not occur (but other perceptually unwelcome transformations could). The adoption of Leonardo's window or a similar optical aid would in fact 
prevent the incorporation into pictures of the kind of visual experience demonstrated in the experiment; unconstrained viewing and lack of awareness of a projection system could lead to divergent perspective being incorporated into pictures.

The perceptual problem at the core of this experiment is clearly that of the visual angle supported by the objects and of the perceived distance between the observer and the objects in question. If the artist chooses to convey his or her impression of an object by conveying to the viewer not the size of projection on a notional screen but the size of the angles supported at his or her eye, then laterally displaced objects will be depicted in divergent perspective. However, an angle on its own does not furnish sufficient information for unambiguous perception of an object. A distance from the object needs to be known (or estimated) before a correct assessment of the true significance of the angle can be made.

In the present experiments subjects were able to see the floor of the laboratory on which the apparatus rested and therefore were aware that the two sets of lights were on a horizontal plane and at different distances from them, yet they matched the two sets of lights in such a way that when viewing them centrally they clearly underestimated the remote distance, and when viewing them laterally they overestimated it; this explanation has the merit of directness.

The distances at which the subjects set the lights under the two conditions can be converted into the angles supported at a hypothetical eye. If this eye is assumed to be $1.6 \mathrm{~m}$ above the floor, it sustains the following angles: frontal viewing: fixed lights $(1 \mathrm{~m}$ apart $)-28 \mathrm{deg}$, mobile lights $(1.16 \mathrm{~m}$ apart $)-15 \mathrm{deg}$; lateral viewing: fixed lights ( $1 \mathrm{~m}$ apart $)-9 \mathrm{deg}$, mobile lights $(0.9 \mathrm{~m}$ apart $)-8 \mathrm{deg}$.

Clearly, therefore, setting of the lights at the geometrically correct distance of $1 \mathrm{~m}$ would, in the case of frontal viewing, increase discrepancy between the angles. In the case of lateral viewing, on the other hand, this relationship does not prevail; on the contrary, setting of the lights at the correct distance apart would reduce the angular discrepancy.

Interpolation of the data obtained offers an analogous explanation for the use of parallel projection wherein receding lines are drawn as neither diverging nor converging but as parallel and at an angle to the horizontal. Such drawings, like drawings showing divergent perspective, are found passim in art. Durer's depiction of a stretcher on which a corpse is resting in his The Joys of the World and the Warning of Death is an instance of the style [see Kurth (1927) figure 15]. Many traditional Japanese drawings use this method of depiction consistently. This method of conveying pictorial depth has an obvious advantage of simplicity of construction; an artist being merely required to draw parallel sloping lines.

These considerations suggest that Moray and Moray (1981, page 702) are in error when, whilst examining Humphrey's (1971) earlier observations on Byzantine art, they state that "since the object is seen obliquely from above, the top is naturally seen as a parallelogram". Our results clearly show this not to be the case; viewed frontally, a rectangular object is 'naturally seen' as a trapezium with the longer of the two frontoparallel sides corresponding to the edges closer to the observer. Displaced laterally it is seen as a trapezium but with the relative sizes of two edges reversed. In an intermediate position it may be seen as a parallelogram.

Therefore perception of a rectangular object as a parallelogram is but one of the equally legitimate possibilities. Furthermore, contrary to Moray and Moray's suggestions, it is not necessary to invoke lack of skill on the part of the ancient copyists to explain divergent perspective.

Topper and Simpson (1981) used Gregory's (1974) Pandora's box to measure perceived depth in four pictures. Two of these pictures are of especial interest because one of them, J Vermeer's The Geographer, embodies convergent perspective, 
whilst the second, a medieval work, Abraham's Vision in the Plains of Mamre and Abraham Entertaining the Angels, from Sta Maria Maggiore in Rome, embodies divergent perspective. Both pictures are also rich in other depth cues. In both pictures, on average, perception of depth was obtained with those elements from which one would expect it on the basis of nonperspective cues (height in the picture, overlap, etc); elements intended to be seen as further away were in fact judged to be further away, although there were some instances of reversal. The mean results and the reversals are both explicable in terms of findings presented here, if one is willing to assume that the viewer decides whether the picture is to be interpreted in accordance with the values of 'central' representation or the rules of 'laterally displaced' representation. When a correct decision is made (and the presence of other concordant depth cues is likely to influence it greatly) 'correct' depth is perceived; when the decision is wrong and, say, a 'laterally displaced' representation is treated as if it were a 'central' representation a reversal takes place.

Topper and Simpson rightly point out that their results are contrary to Goodman's (1968) notion that a picture is a purely conventional denotative object. They further argue that the results, whilst supporting Gombrich's (1960) idea of a picture creating an illusion of space, also cause difficulties for such a notion since divergent perspective is shown to create such an illusion. Gombrich is reported by these authors to have suggested in a private communication that inverse perspective pictures are read rather than seen. Our results suggest that there was no need for such a defence of Gombrich's notion, because divergent perspective is, under specific circumstances, visually legitimate. Thus in our experiments, apart from demonstrating the perceptual legitimacy of inverse perspective, we also show that in drawing visual perceptual paradigms, due attention should be given to the effects of lateral displacement.

Acknowledgement. We thank Miss Fiona Henderson for her assistance with this experiment.

\section{References}

Attneave F, 1954 "Some informational aspects of visual perception" Psychological Review 61 $183-193$

Bartel K, 1958 Perspektywa Malarska volume 2 (Warsaw: Państwowe Wydawnictwo Naukowe) (in Polish)

Carter B A R, 1967 "The use of perspective in Saenredam" Burlington Magazine 109 594-595

Deręgowski J B, 1989 "Geometrical reconstruction of perspective: Bartel's method" Perception $18595-600$

Derȩgowski J B, 1990 Oko i Obraz; Studium Psychologiczne (Warsaw: Paristwowe Wydawnictwo Naukowe)(in Polish)

Doesschate G ten, 1964 Perspective, Fundamentals, Controversials History (Nieuwkoop: B De Graff)

Doesschate G ten, Kylstra J, 1955 "The perception of parallels" Aeromedica 4 115-119

Gillam B, 1981 "False perspectives" Perception 10313-318

Gombrich E H, 1960 Art and Illusion: A Study in the Psychology of Pictorial Representation (Oxford: Phaidon)

Goodman N, 1968 Languages of Art. An Approach to the Theory of Symbols (New York: BobbsMerrill)

Gregory R, 1974 Concepts and Mechanisms of Perception (New York: Scribner)

Humphrey N K, 1971 "Contrast illusions in perspective" Nature (London) 232 91-93

Kemp M, 1984 "Construction and cunning: The perspective of the Edinburgh Saenredam" in Dutch Church Painters; Saenredam's Great Church in Haarlem, in Context Ed. National Gallery of Scotland (Edinburgh: The National Gallery of Scotland) pp 30-37

Kurth W (Ed.), 1927 The Complete Woodcuts of Albrecht Dürer (London: W \& G Foyle)

Moray G, Moray N, 1981 "A note on the basis of reversed perspective" Perception 10 $703-705$

Osborne H, 1981 The Oxford Companion to Art (Oxford: Clarendon Press)

Parker D M, Dereggowski J B, 1990 Perception and Artistic Style (Amsterdam: North-Holland)

Topper D R, Simpson W, 1981 "Depth perception in linear and inverse perspective pictures" Perception $10305-312$

Zajac J L, 1961 "Studies in perspective" British Journal of Psychology 59 333-340 